\title{
DESENVOLVIMENTO E EXIGÊNCIAS TÉRMICAS DE TRICHOGRAMMA PRETIOSUM RILEY, CRIADOS EM DUAS TRAÇAS DO TOMATEIRO ${ }^{1}$
}

\author{
DIRCEU PRATISSOLI² e JOSÉ ROBERTO POSTALI PARRA ${ }^{3}$
}

\begin{abstract}
RESUMO - Estudou-se a biologia de Trichogramma pretiosum em ovos de Phthorimaea operculella (Zeller) e Tuta absoluta (Meyrick) em diferentes temperaturas $\left(18,20,22,25,30\right.$ e $\left.32^{\circ} \mathrm{C}\right), 70 \pm 10 \%$ de umidade relativa e fotofase de 14 horas, com o objetivo de se determinarem as exigências térmicas, bem como o número de gerações durante $\mathrm{o}$ ano. Houve correlação inversa entre a duração do ciclo e o aumento de temperatura na faixa térmica estudada. A razão sexual, no entanto, não foi afetada pela temperatura quando o hospedeiro foi $T$. absoluta. A viabilidade de T. pretiosum, criado em $P$. operculella foi mais afetada que em T. absoluta. A exigência térmica, calculada pelo método da hipérbole, foi maior no tocante a T. absoluta (131,3 graus dia) em relação a P. operculella (120,9 graus dia), e o limiar térmico inferior, respectivamente, 12,98 e $13,53^{\circ} \mathrm{C}$. No que tange a ambas as espécies de traças, a longevidade do parasitóide sempre obedeceu à distribuição de Weibull. O potencial de aumento foi avaliado, em ambas as espécies de traças, com base no número de gerações anuais.
\end{abstract}

Termos para indexação: pragas de plantas, métodos de controle de pragas, controle biológico, Trichogrammatidae.

\section{DEVELOPMENT AND THERMAL REQUIREMENTS OF TRICHOGRAMMA PRETIOSUM RILEY, REARING IN TWO TOMATO LEAFMINER}

\begin{abstract}
The biology of Trichogramma pretiosum in eggs of Phthorimaea operculella (Zeller) and of Tuta absoluta (Meyrick) were studied under different temperatures $\left(18,20,22,25,30,32^{\circ} \mathrm{C}\right)$, $70 \pm 10 \%$ RU and photophase of 14 hours, with the aim of determining the thermal requirements and the number of generations during the year. There was an inverse correlation between the duration of the cycle and the increase of temperature in the thermal zone studied. Nevertheless, the sexual ratio was not affected by the temperature when the host was T. absoluta. The viability of T. pretiosum, reared in P. operculella, was more affected than in T. absoluta. The thermal requirement, calculated by the hyperbole was higher for T. absoluta (131.3 degrees day) in relation to P. operculella (120.9 degrees day), and the thermal threshold was lesser respectively, $12.98^{\circ} \mathrm{C}$ and $13.53^{\circ} \mathrm{C}$. For both species of leafminer, the longevity of parasitoid always obeyed the Weibull's distribution. The increase of the potential was estimated for both species of leafminer, according to the number of annual generations.
\end{abstract}

Index terms: pests of plants, pest control methods, biological control, Trichogrammatidae.

\section{INTRODUÇÃO}

O tomateiro (Lycopersicon esculentum Mill) é atacado por inúmeras pragas e doenças. Entre as pra-

\footnotetext{
${ }^{1}$ Aceito para publicação em 5 de julho de 1999.

${ }^{2}$ Eng. Agrôn., Centro Agropecuário, Universidade Federal do Espírito Santo (UFES), CEP 29500-000 Alegre, ES. E-mail: dirceu@npd.ufes.br

${ }^{3}$ Eng. Agrôn., Dep. de Entomologia, Escola Superior de Agricultura Luiz de Queiroz (ESALQ), Caixa Postal 9, CEP 13418-900 Piracicaba, SP.

E-mail: jrpparra@ carpa.ciagri.usp.br
}

gas, as traças ocupam lugar de destaque, especialmente Tuta absoluta (Meyrick) (Lepidoptera: Gelechiidae), que ataca tanto tomateiro rasteiro (industrial) como estaqueado (de mesa). Phthorimaea operculella (Zeller) (Lepidoptera: Gelechiidae), a traça-da-batatinha, tem sido relatada como praga apenas de tomateiro estaqueado e somente em algumas regiões produtoras. Os prejuízos, dependendo das condições climáticas, especialmente temperatura, chegam a ser totais, pois as traças atacam flores, parênquima foliar, brotos terminais, caule e frutos (Haji, 1992). 
O controle desses insetos tem sido, tradicionalmente, feito principalmente com o uso de inseticidas. Nos últimos anos, especialmente T. absoluta tem-se mostrado resistente a vários produtos químicos. Uma das alternativas neste caso, é o uso de controle biológico através do parasitóide de ovos Trichogramma pretiosum Riley (Hymenoptera: Trichogrammatidae), que já é utilizado com sucesso na Colômbia e no nordeste do Brasil (Petrolina, PE e Juazeiro, BA), através de liberações inundativas, associando-o a produtos biológicos e químicos seletivos (Faria Júnior, 1992).

O gênero Trichogramma vem sendo utilizado em todo o mundo, como agente de controle biológico, pelo fato de ter uma ampla distribuição geográfica, ser altamente especializado e eficiente, ter sido constatado parasitando ovos de pragas de milho, arroz, soja, cana-de-açúcar, sorgo, algodão, beterraba, tomate, florestas, pomares, hortaliças, oliveira, banana, mandioca e ornamentais (Nikonov et al., 1991; Hassan, 1993).

É conhecido que, entre os fatores físicos, a temperatura é o de maior influência sobre aspectos biológicos desse parasitóide, tais como: fecundidade, duração do ciclo de desenvolvimento, razão sexual, viabilidade e longevidade (Harrison et al., 1985; Noldus, 1989).

Além disso, o estudo das exigências térmicas pode auxiliar na seleção de linhagens ou espécies de Trichogramma, na produção massal desse parasitóide, bem como na previsão de liberações no campo (Pratissoli, 1995).

Com o objetivo de se determinarem as exigências térmicas e visando fornecer subsídios à racionalização de liberações de T. pretiosum, estudou-se sua biologia em seis temperaturas constantes, criando-o nas duas espécies de traças que atacam o tomateiro, ou seja, T. absoluta e P. operculella.

\section{MATERIAL E MÉTODOS}

Ovos de P. operculella e T. absoluta foram obtidos das criações mantidas no Laboratório de Biologia do Departamento de Entomologia, da Escola Superior de Agricultura "Luiz de Queiroz", segundo Pratissoli (1995). A coleta dos ovos do primeiro hospedeiro foi feita recortando-se as massas aderidas em disco de papel de filtro. No segundo, a coleta foi feita retirando-se os ovos das folhas do tomate, com um pincel umedecido em água. Para cada umas das traças, 12 cartelas contendo 70 ovos do dia foram isolados em tubos de vidro de $13,0 \times 1,0 \mathrm{~cm}$, fechado com película de plástico de PVC, contendo uma gota de mel puro para alimentação dos adultos de T. pretiosum. Foram liberadas, em cada tubo, sete fêmeas (na proporção de um parasitóide para dez ovos), permitindo um parasitismo por cinco horas, em câmara climatizada, regulada a $25 \pm 1^{\circ} \mathrm{C}, 70 \pm 10 \%$ de umidade relativa (UR) e fotofase de 14 horas. Ao final desse período, as fêmeas foram retiradas sob microscópio estereoscópico, e cada conjunto de tubos foi transferido para câmaras climatizadas, reguladas para as temperaturas de $18,20,22,25,30$ e $32^{\circ} \mathrm{C}, 70 \pm 10 \%$ de UR e fotofase de 14 horas.

Em cada temperatura, foram observados parâmetros biológicos, como a duração do período ovo-adulto, a porcentagem de emergência (viabilidade), a razão sexual e o número de indivíduos por ovo.

Para o cálculo da duração do período ovo-adulto, foram feitas observações diariamente, sempre no mesmo horário, a partir das $17 \mathrm{~h}$

A avaliação da emergência dos parasitóides foi realizada com auxílio de um estilete, sob microscópio estereoscópico, contando-se os ovos dos hospedeiros que apresentavam orifício de saída dos adultos. O sexo dos indivíduos foi determinado através do dimorfismo apresentado pelas antenas (Bowen \& Stern, 1966), com auxílio de um microscópio estereoscópico. O número de indivíduos por ovo foi avaliado por meio da contagem dos adultos que emergiam em cada lote de ovos.

O cálculo do limiar térmico inferior (tb) e da constante térmica $(\mathrm{K})$ foi feito utilizando-se o método da hipérbole (Haddad \& Parra, 1984), com base na duração do período ovo-adulto e nas diferentes temperaturas testadas, para as duas espécies de traças estudadas.

O número provável de gerações de T. pretiosum durante $o$ ano foi calculado em relação às diferentes regiões do Estado de São Paulo, tomando-se como referência as regiões onde se cultiva tomate, com base num mapa de isotermas.

O delineamento experimental utilizado foi inteiramente casualizado, sendo os resultados submetidos à análise de variância, e as médias comparadas pelo teste de Tukey, a $5 \%$ de probabilidade. Os resultados do número de indivíduos por ovo não foram analisados estatisticamente, em razão do número insuficiente de algumas repetições.

\section{RESULTADOS E DISCUSSÃO}

A duração média do desenvolvimento ovo-adulto de $T$. pretiosum referente às duas traças do toma- 
teiro foi afetada significativamente pela temperatura, e ocorreu um aumento na velocidade de desenvolvimento com a elevação da temperatura, nas condições experimentais estudadas (Tabelas 1 e 2). A influência da temperatura sobre a velocidade de desenvolvimento desse parasitóide foi relatada por diversos autores (Calvin et al., 1984; Parra et al., 1991; Sales Júnior, 1992).

Os valores obtidos, no que diz respeito à duração do ciclo desta espécie do parasitóide, assemelhamse aos apresentados por Calvin et al. (1984). Bleicher \& Parra (1989), trabalhando com a mesma faixa de temperatura, com três populações de Trichogramma, observaram, na faixa de $18^{\circ} \mathrm{C} \mathrm{a} 20^{\circ} \mathrm{C}$, que as populações de Piracicaba (SP) e Iguatu (CE) apresentaram uma duração do ciclo superior à obtida neste trabalho, ocorrendo o inverso com a população de Goiânia (GO).
A duração média do ciclo de T. pretiosum, quando criado em ovos de T. absoluta e P. operculella foi muito próxima, nas diferentes temperaturas (Tabelas 1 e 2). Contudo, os menores valores para a duração, foram obtidos sobre o segundo hospedeiro, com exceção da temperatura de $25^{\circ} \mathrm{C}$. Não houve variação na duração do ciclo desse parasitóide, na faixa de $20^{\circ} \mathrm{C}$ a $30^{\circ} \mathrm{C}$, quando mantido sobre os hospedeiros P. operculella e T. absoluta. Butler Junior \& López (1980), estudando o desenvolvimento de T. pretiosum em dois hospedeiros, Sitotroga cerealella (Olivier) e Trichoplusia ni (Huebner), obtiveram resultados semelhantes ao da presente pesquisa. Resultados análogos também foram obtidos por Yu et al. (1984) criando Trichogramma minutum (Riley) em ovos de Anagasta kuehniella (Zeller) e Cydia pomonella (L.), em diferentes condições térmicas. Tais valores, registrados por diferen-

TABELA 1. Duração média do ciclo (ovo-adulto), viabilidade, razão sexual e número de indivíduos por ovo de uma linhagem de Trichogramma pretiosum criada em ovos de Tuta absoluta, em diferentes temperaturas, umidade relativa de $70 \pm 10 \%$ e fotofase de 14 horas ${ }^{1}$.

\begin{tabular}{cccccc}
\hline $\begin{array}{c}\text { Temperatura } \\
\left({ }^{\circ} \mathrm{C}\right)\end{array}$ & $\begin{array}{c}\text { Duração } \\
\text { (dias) }\end{array}$ & $\begin{array}{c}\text { Intervalo de } \\
\text { variação }\end{array}$ & $\begin{array}{c}\text { Viabilidade } \\
(\%)\end{array}$ & $\begin{array}{c}\text { Razão } \\
\text { sexual }\end{array}$ & $\begin{array}{c}\text { Indivíduos/ } \\
\text { ovo }\end{array}$ \\
\hline 18 & $26,47 \pm 0,12 \mathrm{a}$ & $26-27$ & $79,80 \pm 3,17 \mathrm{~d}$ & $0,98 \mathrm{a}$ & 1,00 \\
20 & $17,78 \pm 0,15 \mathrm{~b}$ & $17-18$ & $86,12 \pm 3,50 \mathrm{bcd}$ & $0,97 \mathrm{a}$ & 1,00 \\
22 & $16,32 \pm 0,10 \mathrm{c}$ & $16-17$ & $94,34 \pm 2,76 \mathrm{ab}$ & $1,00 \mathrm{a}$ & 1,00 \\
25 & $10,30 \pm 0,13 \mathrm{~d}$ & $10-12$ & $96,21 \pm 0,74 \mathrm{a}$ & $0,99 \mathrm{a}$ & 1,00 \\
30 & $7,50 \pm 0,09 \mathrm{e}$ & $7-9$ & $88,97 \pm 1,85 \mathrm{abc}$ & $0,98 \mathrm{a}$ & 1,00 \\
32 & $7,11 \pm 0,06 \mathrm{f}$ & $7-8$ & $83,37 \pm 2,26 \mathrm{~cd}$ & $0,98 \mathrm{a}$ & 1,00 \\
\hline
\end{tabular}

1 Médias seguidas da mesma letra, na vertical, não diferem estatisticamente entre si, pelo teste de Tukey, a 5\% de probabilidade.

TABELA 2. Duração média do ciclo (ovo-adulto), viabilidade, razão sexual e número de indivíduos por ovo de uma linhagem de Trichogramma pretiosum criada em ovos de Phthorimaea operculella, em diferentes temperaturas, umidade relativa de $70 \pm 10 \%$ e fotofase de 14 horas ${ }^{1}$.

\begin{tabular}{ccclcc}
\hline $\begin{array}{c}\text { Temperatura } \\
\left({ }^{\circ} \mathrm{C}\right)\end{array}$ & $\begin{array}{c}\text { Duração } \\
\text { (dias) }\end{array}$ & $\begin{array}{c}\text { Intervalo de } \\
\text { variação }\end{array}$ & $\begin{array}{c}\text { Viabilidade } \\
(\%)\end{array}$ & $\begin{array}{c}\text { Razão } \\
\text { sexual }\end{array}$ & $\begin{array}{c}\text { Indivíduos/ } \\
\text { ovo }\end{array}$ \\
\hline 18 & $25,80 \pm 0,13 \mathrm{a}$ & $24-28$ & $92,38 \pm 0,78 \mathrm{~d}$ & $0,83 \mathrm{~b}$ & 1,05 \\
20 & $17,28 \pm 0,06 \mathrm{~b}$ & $17-20$ & $90,38 \pm 0,84 \mathrm{bcd}$ & $0,88 \mathrm{ab}$ & 1,15 \\
22 & $15,85 \pm 0,05 \mathrm{c}$ & $15-17$ & $98,39 \pm 0,43 \mathrm{ab}$ & $0,89 \mathrm{ab}$ & 1,11 \\
25 & $10,82 \pm 0,07 \mathrm{~d}$ & $10-13$ & $97,51 \pm 0,36 \mathrm{a}$ & $0,99 \mathrm{a}$ & 1,24 \\
30 & $7,08 \pm 0,04 \mathrm{e}$ & $7-9$ & $95,15 \pm 0,77 \mathrm{abc}$ & $0,96 \mathrm{ab}$ & 1,14 \\
32 & $6,61 \pm 0,05 \mathrm{f}$ & $6-8$ & $88,02 \pm 1,52 \mathrm{~cd}$ & $0,92 \mathrm{ab}$ & 1,18 \\
\hline
\end{tabular}

${ }^{1}$ Médias seguidas da mesma letra, na vertical, não diferem estatisticamente entre si, pelo teste de Tukey, a 5\% de probabilidade 
tes autores, indicam que a variação do ciclo de Trichogramma, em determinada faixa de temperatura, depende da adaptação da espécie ou linhagem e do hospedeiro utilizado.

A viabilidade de T. pretiosum, criado em ovos de T. absoluta e P. operculella, foi afetada pela variação da temperatura (Tabelas 1 e 2). Esses resultados estão de acordo com os obtidos por Parra et al. (1991), Sales Júnior (1992), Tironi (1992); Cônsoli \& Parra (1995a, 1995b). A porcentagem de emergência desse parasitóide, criado em ambas as traças do tomateiro, foi maior na faixa entre $22^{\circ} \mathrm{C}$ e $30^{\circ} \mathrm{C}$. No entanto, a emergência em temperaturas na faixa de $22^{\circ} \mathrm{Ca} 25^{\circ} \mathrm{C}$ apresentou diferenças estatisticamente significativas em relação às temperaturas extremas, tanto em ovos de T. absoluta, quanto em P. operculella $(18,20 \mathrm{e}$ $32^{\circ} \mathrm{C}$ ) (Tabelas $1 \mathrm{e} 2$ ). Anunciada (1983), trabalhando com oito linhagens de Trichogramma, observou que a faixa de temperatura de $20^{\circ} \mathrm{Ca} 30^{\circ} \mathrm{C}$ proporcionou as melhores taxas de emergência. Harrison et al. (1985) observaram essa mesma faixa ótima de temperatura $\left(22^{\circ} \mathrm{Ca} 25^{\circ} \mathrm{C}\right)$ para emergência de $T$. pretiosum. Sales Júnior (1992) e Cônsoli \& Parra (1995a, 1995b) verificaram que, para Trichogramma galloi Zucchi, a taxa de emergência sofreu redução na temperatura de $18^{\circ} \mathrm{C}$. Tironi (1992) também observou que temperaturas inferiores $\mathrm{a} 20^{\circ} \mathrm{C}$ afetaram a porcentagem de emergência de Trichogramma atopovirilia Oatman \& Platner e T. pretiosum. Embora se tenha observado que nas temperaturas extremas à faixa ótima tenham ocorrido reduções na taxa de emergência, Bleicher \& Parra (1989) não encontraram tal variação na viabilidade de três populações de Trichogramma, mesmo em temperaturas extremas. O hospedeiro também interferiu na viabilidade desse parasitóide (Tabelas $1 \mathrm{e}$ 2). T. pretiosum criado em ovos de T. absoluta, nas diferentes temperaturas, apresentou uma taxa de emergência sempre menor quando comparado com aquele criado em ovos de $P$. operculella.

Essas variações podem ser devidas às características do ovo de $P$. operculella, que em diferentes condições térmicas não perde o seu aspecto normal, mantendo-o ao longo do tempo, fato não observado em T. absoluta; o efeito dessa perda da característica do ovo, em função da temperatura, tem sido relatada por diversos autores (Parra et al., 1991; Tironi, 1992) como responsável pela variação da emergência de espécies de Trichogramma.
A razão sexual do parasitóide foi afetada pela temperatura quando criada sobre P. operculella (Tabela 2), havendo diferença estatisticamente significativa entre os valores obtidos na temperatura de $25^{\circ} \mathrm{C} \mathrm{a}$ $18^{\circ} \mathrm{C}$. Com relação aos parasitóides criados sobre T. absoluta, não se observou interferência da temperatura na razão sexual (Tabela 1). Lund (1938) observou que o decréscimo da temperatura proporcionou uma diminuição na razão sexual, havendo, portanto, um efeito diferencial da temperatura sobre sexos. Butler Junior \& López (1980), trabalhando com três populações de T. pretiosum, verificaram que para uma delas havia ocorrência maior de fêmeas em temperaturas elevadas. Brun et al. (1981) afirmaram que, em espécies com reprodução deuterótoca, criadas a $21^{\circ} \mathrm{C}$, a ocorrência de machos é rara. Russo \& Voegelé (1982), estudando quatro espécies de Trichogramma, em diferentes temperaturas, constataram que duas delas apresentavam maior número de fêmeas nas temperaturas extremas $\left(13^{\circ} \mathrm{C} \mathrm{e} 30^{\circ} \mathrm{C}\right)$. Bleicher \& Parra (1989) observaram que, nas três populações de Trichogramma estudadas, a razão sexual não foi afetada pela variação da temperatura, o que coincide com os resultados de Tironi (1992). Parra et al. (1991), trabalhando com T. galloi, também observaram que a razão sexual não é afetada pela temperatura. É evidente que a temperatura não é fator determinante do sexo (Stouthamer, 1991; Stouthamer et al., 1993); entretanto, ela pode provocar efeitos diferenciais, em ambos os os sexos, levando a alteração à razão sexual, da forma como ela é determinada em estudos biológicos de laboratório. A linhagem desse parasitóide, quando criado sobre a traça T. absoluta, apresentou uma razão sexual superior à traça P. operculella, em todas as temperaturas estudadas (Tabelas 1 e 2).

O número de parasitóides emergidos por ovo de T. absoluta foi sempre um em todas as temperaturas estudadas (Tabela 1). Em ovos de P. operculella, esse número variou, dependendo da temperatura, sendo sempre superior à de um indivíduo por ovo (Tabela 2). O maior número de parasitóides por ovo foi obtido quando este inimigo natural foi mantido a $25^{\circ} \mathrm{C}$. Observou-se uma tendência de redução deste número na temperatura de $18^{\circ} \mathrm{C}$. Esse fato também foi constatado por Bleicher \& Parra (1989) em duas populações de Trichogramma (Piracicaba, SP e Goiânia, GO). 
Em função da velocidade de desenvolvimento nas diferentes temperaturas $\left(18,20,22,25,30\right.$ e $\left.32^{\circ} \mathrm{C}\right)$, no período ovo-adulto, pôde-se determinar o limite térmico inferior de desenvolvimento (tb), e a constante térmica $(\mathrm{K})$ de $T$. pretiosum nos dois hospedeiros. Foram determinados os respectivos valores de $12,98^{\circ} \mathrm{C}$ e 131,3 graus dia, quando criado sobre T. absoluta, e de, $13,53^{\circ} \mathrm{C}$ e 120,9 graus dia, quando mantido sobre $P$. operculella (Tabela 3 ).

Comparando os valores de tb e $\mathrm{K}$, obtidos neste trabalho (Tabela 3), com os apresentados por outros autores, para diferentes linhagens de $T$. pretiosum (Tabela 4), constatou-se que a temperatura base obtida das duas traças do tomateiro foi ligeiramente superior à maioria dos valores citados na literatura, tendo como conseqüência uma constante térmica inferior aos resultados apresentados nas referidas citações (Tabelas 3 e 4). Os altos valores de $\mathrm{R}^{2}$ obti-

TABELA 3. Temperatura base (tb), constante térmica (K) e coeficiente de determinação $\left(\mathbf{R}^{2}\right)$ de Trichogramma pretiosum criado sobre as duas traças em condições de laboratório, com umidade relativa de $70 \pm 10 \%$ e fotofase de 14 horas.

\begin{tabular}{lccc}
\hline Espécie & $\begin{array}{c}\mathrm{tb} \\
\left({ }^{\circ} \mathrm{C}\right)\end{array}$ & $\begin{array}{c}\mathrm{K} \\
\text { (graus dia) }\end{array}$ & $\begin{array}{c}\mathrm{R}^{2} \\
(\%)\end{array}$ \\
\hline T. absoluta & 12,98 & 131,3 & 98,6 \\
P. operculella & 13,53 & 120,9 & 99,0 \\
\hline
\end{tabular}

dos atestam a confiabilidade dos resultados alcançados (Tabela 3). Tomando-se por base os dados da presente pesquisa (tb e K) (Tabela 3 ) e os registrados por outros autores (Tabela 4), constatam-se variações decorrentes da linhagem de T. pretiosum e do hospedeiro de criação. Orphanides \& Gonzalez (1971), Butler Junior \& López (1980), Goodenough et al. (1983), Calvin et al. (1984), Bleicher \& Parra (1990) e Tironi (1992), avaliaram a tb e $\mathrm{K}$ de $T$. pretiosum em diversos hospedeiros, obtendo valores na faixa de 9,6 a 12,8 e de 125,6 a 183,3, respectivamente (Tabela 4). Essas variações também foram verificadas em outras espécies de Trichogramma (Lund, 1934; Stern \& Bowen, 1963; Bowen \& Stern, 1966; Russo \& Voegelé, 1982; Yu et al., 1984; Zhang, 1984; Hirashima et al., 1990).

A influência da espécie, linhagem e hospedeiro nas exigências térmicas desse parasitóide tem sido relatada por diversos autores (Orphanides \& Gonzalez, 1971; Butler Junior \& López, 1980; Yu et al. 1984; Hirashima et al., 1990; Sales Júnior, 1992; Tironi, 1992). Por esse motivo, as pesquisas devem ser direcionadas para obtenção de um hospedeiro alternativo com características semelhantes à da praga que se quer controlar, para que o parasitóide expresse todo seu potencial no campo.

A estimativa anual do número de gerações de T. pretiosum sobre as duas traças aumentou significativamente com a elevação da temperatura, chegando a 33,4 gerações no primeiro hospedeiro e 34,6 gerações no segundo, em condições ótimas (Tabela 5).

TABELA 4. Temperatura base (tb), constante térmica (K) e coeficiente de determinação $\left(R^{2}\right)$ de várias linhagens de Trichogramma pretiosum, em diferentes hospedeiros, tomando-se por base os dados de diversos autores. Os cálculos foram feitos pelo método da hipérbole.

\begin{tabular}{llcccc}
\hline Espécie & Hospedeiro & tb $\left({ }^{\circ} \mathrm{C}\right)$ & $\mathrm{K}$ (graus dia) & $\mathrm{R}^{2}(\%)$ & Autores \\
\hline T. pretiosum & Heliothis virescens & 10,2 & 164,6 & 95,6 & Goodenough et al. (1983) \\
T. pretiosum & Sitotroga cerealella & 10,7 & 174,4 & 95,9 & Goodenough et al. (1983) \\
T. pretiosum & Anagasta kuehniella & 10,0 & 169,4 & 95,0 & Goodenough et al. (1983) \\
T. pretiosum & Galeria mellonella & 11,4 & 183,3 & 99,4 & Goodenough et al. (1983) \\
T. pretiosum & Diatraea grandiosella & 9,9 & 158,7 & 91,2 & Calvin et al. (1984) \\
T. pretiosum & Sitotroga cerealella & 11,5 & 125,6 & 96,0 & Orphanides \& Gonzalez (1971) \\
T. pretiosum & Sitotroga cerealella & 12,2 & 128,7 & 99,6 & Butler Junior \& López (1980) \\
T. pretiosum & Trichoplusia ni & 11,3 & 131,5 & 99,3 & Butler Junior \& López (1980) \\
T. pretiosum (pop. Iguatu) & Anagasta kuehniella & 12,8 & 133,2 & 96,3 & Bleicher \& Parra(1990) \\
T. pretiosum (pop. Goiânia) & Anagasta kuehniella & 11,9 & 131,9 & 97,8 & Bleicher \& Parra(1990) \\
T. pretiosum & Anagasta kuehniella & 9,6 & 154,8 & 99,4 & Tironi (1992)
\end{tabular}


TABELA 5. Número provável de gerações de Trichogramma pretiosum durante o ano, criadas em ovos de Tuta absoluta e Phthorimaea operculella, em regiões do Estado de São Paulo de isotermas semelhantes.

\begin{tabular}{ccc}
\hline Isotermas $\left({ }^{\circ} \mathrm{C}\right)$ & \multicolumn{2}{c}{$\mathrm{N}^{\mathrm{o}}$ de gerações } \\
\cline { 2 - 3 } & T. absoluta & P. operculella \\
\hline 20 a 21 & 19,5 a 22,3 & 19,5 a 22,6 \\
21 a 22 & 22,3 a 25,1 & 22,6 a 25,6 \\
22 a 23 & 25,1 a 27,8 & 25,6 a 28,6 \\
23 a 24 & 27,8 a 30,6 & 28,6 a 31,6 \\
24 a 25 & 30,6 a 33,4 & 31,6 a 34,6 \\
$>25$ & $>33,4$ & $>34,6$ \\
\hline
\end{tabular}

Nas isotermas compreendidas na faixa de $23^{\circ} \mathrm{Ca} 25^{\circ} \mathrm{C}$, o parasitóide, em ovos de $P$. operculella, apresentou um aumento de gerações variável de 0,8 a $1,2 \mathrm{em}$ relação a T. absoluta. Tal alongamento do ciclo em T. absoluta reforça a hipótese de esta espécie não ser um hospedeiro favorável para a multiplicação de T. pretiosum em relação a $P$. operculella.

As determinações das exigências térmicas de T. pretiosum sobre os dois hospedeiros utilizados poderão auxiliar na elaboração dos modelos de controle das duas traças, bem como auxiliar na produção massal do parasitóide em laboratório (Parra, 1994).

\section{CONCLUSÕES}

1. A velocidade de desenvolvimento aumenta com a elevação térmica em ambas as traças.

2. A variação do ciclo de T. pretiosum, na faixa térmica em estudo, decorre da linhagem e do hospedeiro utilizado.

3. As melhores temperaturas para o desenvolvimento e viabilidade de $T$. pretiosum, quando criados em T. absoluta e $P$. operculella, são $22^{\circ} \mathrm{C}$ e $25^{\circ} \mathrm{C}$;

4. A maior viabilidade do parasitóide sempre ocorre sobre o hospedeiro $P$. operculella, em todas as temperaturas estudadas.

5. Com base nas exigências térmicas do parasitóide, o número provável de gerações, em re- giões com isotermas semelhantes, é sempre superior no hospedeiro $P$. operculella.

6. As exigências térmicas auxiliam na produção massal do parasitóide, bem como na previsão de liberações no campo.

7. P. operculella é mais adequada ao desenvolvimento de T. pretiosum em relação a T. abosluta.

\section{REFERÊNCIAS}

ANUNCIADA, L. A escolha de um oófago Trichogramma para o controle de Mythimma unipuncta. Ponta Delgada : Universidade dos Açores, 1983. 208p. Tese de Doutorado.

BLEICHER, E.; PARRA, J.R.P. Espécies de Trichogramma parasitóides de Alabama argillacea I. Biologia de três populações. Pesquisa Agropecuária Brasileira, Brasília, v.24, n.8, p.929940, ago. 1989.

BLEICHER, E.; PARRA, J.R.P. Espécies de Trichogramma parasitóides de Alabama argillacea. III. Determinação das exigências térmicas de três populações. Pesquisa Agropecuária Brasileira, Brasília, v.25, n.2, p.215-219, fev. 1990.

BOWEN, W.R.; STERN, V.M. Effect of temperature on the production of males and sexual mosaics in a uniparental race of Trichogramma semifumatum (Hymenoptera: Trichogrammatidae). Entomological Society of America Annals, Lanham, v.59, n.4, p.823-834, 1966.

BRUN, P.G.; MARQUES, J., MORAES, G.W.G. Estudo da influência da temperatura no aparecimento de machos em espécies deuterótocas de Trichogramma sp. (Hym.: Trichogrammatidae). In: CONGRESSO BRASILEIRO DE ENTOMOLOGIA, 7., 1981, Fortaleza. Anais. Fortaleza : Sociedade Entomológica do Brasil, 1981. p.143-144

BUTLER JUNIOR, G.D.; LÓPEZ, J.D. Trichogramma pretiosum. Development in two hosts in relation to constant and fluctuating temperatures. Entomological Society of America Annals, Lanham, v.73, n.6, p.671-673, 1980.

CALVIN, D.D.; KNAPP, M.C.; WELCH, S.M.; POSTON, F.L.; ELZINGA, R.J. Impact of environmental factors on Trichogramma pretiosum reared on southwestern corn borer eggs. 
Environmental Entomology, Lanham, v.13, n.3, p.774-780, 1984.

CÔNSOLI, F.L.; PARRA, J.R.P. Effects of constant and alternating temperatures on Trichogramma galloi Zucchi (Hym.: Trichogrammatidae) biology. I. Developmental and thermal requirements. Journal of Applied Entomology, Berlin, v.119, p.415-418, 1995a

CÔNSOLI, F.L.; PARRA, J.R.P. Effects of constant and alternating temperatures on Trichogramma galloi Zucchi (Hym.: Trichogrammatidae) biology. II. Parasitism capacity and longevity. Journal of Applied Entomology, Berlin, v.119, p.667-670, $1995 \mathrm{~b}$

FARIA JÚNIOR, P.A.J. Controle biológico da traça do tomateiro pela "FRUTINOR". In: SIMPÓSIO DE CONTROLE BIOLÓGICO, 3., 1992, Águas de Lindóia. Anais. Águas de Lindóia : Embrapa, 1992. p.61-63.

GOODENOUGH, J.L.; HARTSTACK, A.W.; KING, E.G. Developmental models for Trichogramma pretiosum (Hymenoptera: Trichogrammatidae) reared on four hosts. Journal of Economic Entomology, Lanham, v.76, n.5, p.1095-1102, 1983.

HADDAD, M.L.; PARRA, J.R.P. Métodos para estimar os limites térmicos e a faixa ótima de desenvolvimento das diferentes fases do ciclo evolutivo de insetos. Piracicaba : FEALQ, 1984. 12p. (Agricultura e Desenvolvimento)

HAJI, F.N.P. Histórico e situação atual da traça do tomateiro nos perímetros irrigados ao Submédio São Francisco. In: SIMPÓSIO DE CONTROLE BIOLÓGICO, 3., 1992, Águas de Lindóia. Anais. Águas de Lindóia : Embrapa, 1992. p.57-59.

HARRISON, W.W.; KING, E.G.; OUZTS, J.D Development of Trichogramma exiguum and T. pretiosum at five temperature regimes. Environmental Entomology, Lanham, v.14, n.2, p.118-121, 1985.

HASSAN, S.A. The mass rearing and utilization of Trichogramma to control lepidopterous pests: Achievements and outlook. Pesticide Science, Chichester, v.37, p.387-391, 1993

HIRASHIMA, Y.; MIURA, K.; MIURA, T.; HASEGAWA, T. Studies on the biological control of the diamondback moth, Plutella xylostella (Linnaeus) IV. Effect of temperature on the development of the egg parasitoids Trichogramma chilonis and Trichogramma ostriniae. Kyushu University Faculty of Agriculture Science Bulletin, Fukuoka, v.44, n.3, p.81-87, 1990.

LUND, H.O. Some temperature and humidity relations of two races of Trichogramma minutum Riley (Hym. Chalcididae). Entomological Society of America Annals, Lanham, v.27, p.324-340, 1934.

LUND, H.O. Studies on longevity and productivity in Trichogramma evanescens. Journal of Agricultural Research, London, v.56, n.6, p.421439,1938

NIKONOV, P.V.; LEBEDEV, G.L.; STARTCHEVSKY, I.P. Trichogramma production in the USSR. In: INTERNATIONAL SYMPOSIUM ON TRICHOGRAMMA AND OTHER EGG PARASITOIDS, 3., 1990, San Antonio. Proceedings. Paris : INRA, 1991. p.151-152.

NOLDUS, L.P.J.J. Semiochemicals, foraging behaviour and quality of entomophagous insects for biological control. Journal of Applied Entomology, Berlin, v.108, p.425-451, 1989

ORPHANIDES, G.M.; GONZALEZ, D. Fertility and life table studies with Trichogramma pretiosum and T. retorridum (Hymenoptera: Trichogrammatidae). Entomological Society of America Annals, Lanham, v.64, n.4, p.824-834, 1971.

PARRA, J.R.P. Técnicas de criação de insetos para programas de controle biológico. Piracicaba : FEALQ, 1994. 192p.

PARRA, J.R.P.; ZUCCHI, R.A.; SILVEIRA NETO, S.; HADDAD, M.L. Biology and thermal requirements of Trichogramma galloi Zucchi and T. distinctum Zucchi, on two alternative hosts. In: INTERNATIONAL SYMPOSIUM ON TRICHOGRAMMA AND OTHER EGG PARASITOIDS, 3., 1990, San Antonio. Proceedings. Paris : INRA, 1991. p.81-84

PRATISSOLI, D. Bioecologia de Trichogramma pretiosum Riley, 1879, nas traças Scrobipalpuloides absoluta (Meyrick, 1917) e Phthorimaea operculella (Zeller, 1873), em tomateiro. Piracicaba : ESALQ, 1995. 135p. Tese de Doutorado.

RUSSO, J.; VOEGELÉ, J. Influence de la température sur quatre espéces de Trichogrammes (Hym.: Trichogrammatidae) parasites de la pyrale du mais,

Pesq. agropec. bras., Brasília, v.35, n.7, p.1281-1288, jul. 2000 
Ostrinia nubilalis Hubn. (Lep.: Pyralidae). I. Reproduction et survie. Agronomie, Paris, v.2, n.6, p.517-524, 1982

SALES JÚNIOR, O. Bioecologia de Trichogramma galloi Zucchi, 1988 no hospedeiro natural Diatraea saccharalis (Fabricius, 1794) e em hospedeiros alternativos. Piracicaba : ESALQ, 1992. 97p. Tese de Doutorado.

STERN, V.M.; BOWEN, W. Ecological studies of Trichogramma semifumatum with notes on Apanteles medicaginis, and their suppression of Colias eurytheme in Southern California. Entomological Society of America Annals, Lanham, v.56, p.358372,1963 .

STOUTHAMER, R. Effectiveness of several antibiotics in reverting thelytoky to arrhenotoky in Trichogramma spp. In: INTERNATIONAL SYMPOSIUM ON TRICHOGRAMMA AND OTHER EGG PARASITOIDS, 3., 1990, San Antonio. Proceedings. Paris, INRA, 1991. p.119-122.
STOUTHAMER, R.; BREEUWER, J.A.J.; LUCK, R.F.; WERREN, J.H. Molecular identification of microorganisms associated with parthenogenesis. Nature, London, v.361, n.7, p.66-68, 1993.

TIRONI, P. Aspectos bioecológicos de Trichogramma pretiosum Riley, 1879 e Trichogramma atopovirilia Oatman \& Platner, 1983 (Hym.: Trichogramnmatidae), como agentes de controle biológico de Helicoverpa zea (Boddie, 1850) (Lep.: Noctuidae). Lavras : ESAL, 1992. 72p. Dissertação de Mestrado.

YU, D.S.K.; HAGLEY, A.C.; LAING, J.E. Biology of Trichogramma minutum Riley collected from apples in Southern Ontario. Environmental Entomology, Lanham, v.13, n.5, p.1324-1329, 1984.

ZHANG, Y.J. Influence of temperature on the development and emergence of Trichogramma spp. Acta Entomologica Sinica, Beijing, v.27, n.1, p.28-38, 1984 